\title{
Evaluation of floating car technologies for travel time estimation
}

\author{
Xiaobo LIU ${ }^{1,2^{*}}$, Steven CHIEN ${ }^{3}$, Kitae $\mathrm{KIM}^{3}$ \\ 1. Jacobs Engineering Group, Department of Planning, Morristown, New Jersey 07962, USA \\ 2. School of Transportation and Logistics, Southwest Jiaotong University, Chengdu 610031, China \\ 3. Department of Civil and Environmental Engineering, New Jersey Institute of Technology, Newark, New Jersey 07102, USA
}

\begin{abstract}
Travel times have been traditionally estimated from data collected by roadway sensors. Recently, new technologies, such as cell phone tracking, license plate matching, automatic vehicle identifications and video detection, are employed for this purpose. In this study, the data collected by TRANSMIT readers, Bluetooth sensors, and INRIX are assessed by comparing each to the "ground truth" travel times collected by probe vehicles carrying GPS-based navigation devices. Travel times of probe vehicles traveling on the study segment of I-287 in New Jersey were collected in 2009. Statistical measures, such as standard deviation, average absolute speed error, and speed error bias, were used to make an in-depth analysis. The accuracy of each travel time estimation method is analyzed. The data collected by Bluetooth sensors and the TRANSMIT readers seem more consistent with the ground true data, and slightly outperform the data reported by INRIX. This study established a procedure for analyzing the accuracy of floating car data (FCD) collected by different technologies.
\end{abstract}

Key words: floating car technology; travel time estimation; statistical analysis

(C) 2012 JMT. All rights reserved.

\section{Introduction}

\begin{abstract}
A ccording to the US Department of Transportation, Intelligent Transport System (ITS) improves transportation safety and mobility and enhances productivity through the integration of advanced communication technologies into the transportation infrastructure and in vehicles. In the advent of ITS, a broad range of communication technologies, including wireless communication, computational technologies, floating car data (FCD), sensing technologies, inductive loop detection, and video vehicle detection are applied for real-time data collection, processing, and management [1]. The goals of real-time data capture and management research is to systematically capture real-time, multimodal data from connected vehicles, devices, and infrastructure and to develop data environments that enable integration of high-quality data from multiple sources for transportation management and performance measures. In the application of FCD, effective methods were developed to determine instantaneous and predict future traffic conditions for disseminating timely information to road users.
\end{abstract}

\footnotetext{
Received Dec. 18, 2011; revision accepted Feb. 6, 2012

*Corresponding author. E-mail: xbliu2010@swjtu.cn (X.B. Liu)

(C) 2012 JMT. All rights reserved

doi: 10.3969/j.issn.2095-087X.2012.01.008
}

\section{Literature review}

The FCD technology employs probe vehicles as agents for collecting traffic data, such as travel speed and time. As discussed in "The Travel Time Data Collection Handbook", the technologies of collecting the probe vehicle data were classified into five categories: signpost-based automatic vehicle location (AVL), automatic vehicle identification (AVI), ground-based radio navigation, cellular geo-location, and GPS. Wikipedia defined FCD into three categories: floating cellular data, electronic toll collection (ETC) and GPS-based. The FCD technology has been widely implemented for travel time collection in past decades. For example, a signpostbased system, typically used by transit agencies for tracking bus locations, relies on transponders attached to roadside signposts. AVL systems and automatic passenger counter (APC) systems are applied for tracking dynamic information, such as vehicle locations, travel times, and arrival times [2]. With ETC on the eight bridges crossing San Francisco Bay Area, Wright and Dahlgren [3] developed a low cost system for measuring travel times on bridges and roads. By using traffic monitoring systems based on wireless location technology (WLT), Fontaine et al. [4] developed a method to estimate sampling parameters based on localized traffic conditions in the network. The proposed sampling strategy effectively improved speed estimation. 
The advantages of utilizing FCD include low cost per unit of data, data continuity, more coverage, automated data collection with standardized electronic format, all weather conditions, and no disruption of traffic. However, the disadvantages may include high startup costs, dependence upon market penetration, permanent infrastructure, and privacy concerns. Successful applications of FCD in ITS [5-13] including dynamic routing guidance, variable message sign (VMS), congestion control strategy, incident detection and management, and transportation modeling applications, are greatly dependent on the accuracy and consistency of real-time information, such as travel time.

In general, the approaches of using FCD for travel time estimation can be classified into two streams. The first one is to locate pairs of sensors at fixed check points to track probe vehicles passing by. The second one discretely transmits the location of probe vehicles via information networks, such as GPS-based systems. Both approaches require a unique identification number (ID) for each probe vehicle so that its presence can be identified. The principle of FCD is to track the probe vehicles based on unique IDs as opposed to traditional sensing technologies (i.e. loop detectors) detecting traffic without identifying individual vehicles.

\section{Objective}

In this study four floating car technologies applied to approximating travel time are evaluated and discussed, which include data collected by Co-Pilot devices (GPSbased), Bluetooth sensors, TRANSMIT, and INRIX.

(1) Co-Pilot data is collected with probe vehicles carrying a GPS navigation device and positional information can be transmitted at 0.3 -second interval to a server for travel time estimation. The GPS-based technology can detect the positions of probe vehicles with higher accuracy compared to those collected by other floating car technologies [14]. In addition, vehicles equipped with Co-Pilot devices are "active" probe vehicles to be flexibly dispatched to collect travel time information for specific corridors during specific time periods. As a result, this data can be treated as "ground truth" in this analysis.

(2) Bluetooth sensors, installed in the roadside of transportation network, collect travel time of vehicles equipped with Bluetooth devices. When a Bluetooth enabled vehicle, mostly Bluetooth-enabled mobile phone, is detected by a pair of sensors, a unique ID in each device (called media access control address, or MAC address) and the time of detection are recorded. These MAC addresses are used for determining speed and travel time information for all Bluetooth enabled vehicle. Observations of multiple vehicles may provide accurate estimates of traffic conditions, such as a number of travel time samples between a pair of Bluetooth sensors.
(3) The TRANSMIT system (TRANSCOM's system for managing incidents $\&$ traffic) uses vehicles equipped with electronic toll-collection tags (E-ZPass) as anonymous probes for travel time collection. Once E-ZPass vehicles pass transponder readers installed roadside, the tags are detected by successive roadside readers, thereby revealing travel time and speed which can be used to determine traffic flow characteristics. By comparing with real-time and historical data, TRANSMIT can detect incidents and alert operators for emergent responses [15].

(4) The INRIX database collects information about roadway speeds from 5 million trucks, delivery vans and other fleet vehicles equipped with GPS devices as well as consumer cellular GPS-based devices. This system constitutes a hybrid FCD which integrates GPS-based FCD and fixed sensor data to provide travel time data for major freeways, highways and arterials across the United States. The initial INRIX system spans from New Jersey to North Carolina covering approximately 1500 center line miles of freeways and 1000 center line miles of major arterials. Currently, INRIX has a broad range of real-time, predictive and historical traffic flow services, including the new INRIX Nationwide Traffic Alerts, providing alerts on over 100000 miles of freeways and the entire interstate highway system. The INRIX Smart Dust Network and INRIX Traffic Fusion Engine seem capable of providing a unique platform and estimate travel time by aggregating and blending traffic data from a variety of sources including over 750000 GPS-enabled vehicles and traditional road sensors for the I-95 Coalition.

This study aims to assess the accuracy of various floating car technologies for travel time estimation. The data collected by these technologies including TRANSMIT roadside readers, Bluetooth sensors, and INRIX were compared with ground truth travel times collected by probe vehicles carrying Co-Pilot devices. Statistical measures are used to make an in-depth comparison.

\section{Case study}

The Interstate I-287 in New Jersey was selected as a case study. In order to conduct a comparative analysis, FCD collected by each technology should cover the same route link and the same time period. The INRIX system utilizes an emerging industry convention known as "traffic message channel (TMC) location codes" to integrate disaggregated data by road link. Four TMC links from the INRIX database were selected and accordingly Bluetooth sensors were located at each TMC link boundary so that the travel time on the same links can be collected by Bluetooth sensors along the study I287. The Bluetooth sensors were installed along a 15mile section of I-287 (see Fig. 1). Probe vehicles with 
Co-Pilot, a GPS-based navigation device, were dispatched to collect travel time data along these links.

With Co-Pilot, travel time samples were collected by a number of probe vehicles traveling on a link of Interstate I-287 in New Jersey between April 6 and April 17, 2009. The Co-Pilot data were processed and used to compare travel time data approximated by the TRANSMIT roadside readers, Bluetooth sensors and the INRIX database. The Bluetooth sensors were installed just over the study I-287 from April 8 to April 23, 2009. The study I-287 is also covered by six TRANSMIT readers which divide the study I-287 into 5 links. To fully cover the TRANSMIT and Bluetooth links, the probe vehicles were dispatched between the start and end locations shown in Fig. 2. The daily study time period (7:00 A.M. to 10:00 A.M.) of Co-Pilot data were divided into twelve 15-minute intervals. Two probe vehicles were dispatched per time interval.

TRANSMIT, INRIX, and Bluetooth dataset were logged in the database indexed by time point. TRANSMIT data is clearly defined as an interval of time occurring before the reported time point. INRIX and Bluetooth data lack clear definitions, however, as the data could represent an interval of time either preceding or succeeding the reported time point. To mitigate this confusion, the estimated speed was based on the number of vehicles passing the study link within an interval before the time point reporting the speed. For example, a 5-minute mean speed at 7:05 A.M. was estimated based on the detected vehicles between 7:00 A.M. and 7:05 A.M.

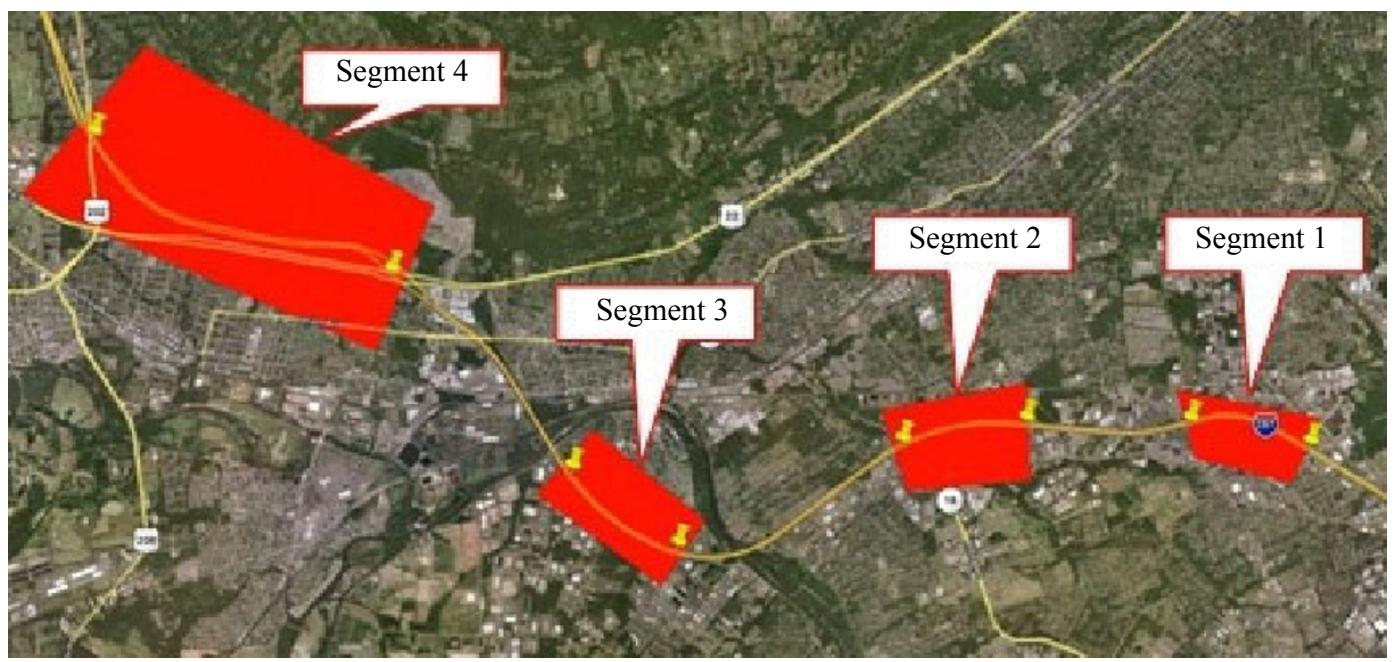

Fig. 1 Locations of Bluetooth Sensors on the study I-287 (depicted by red boxes)

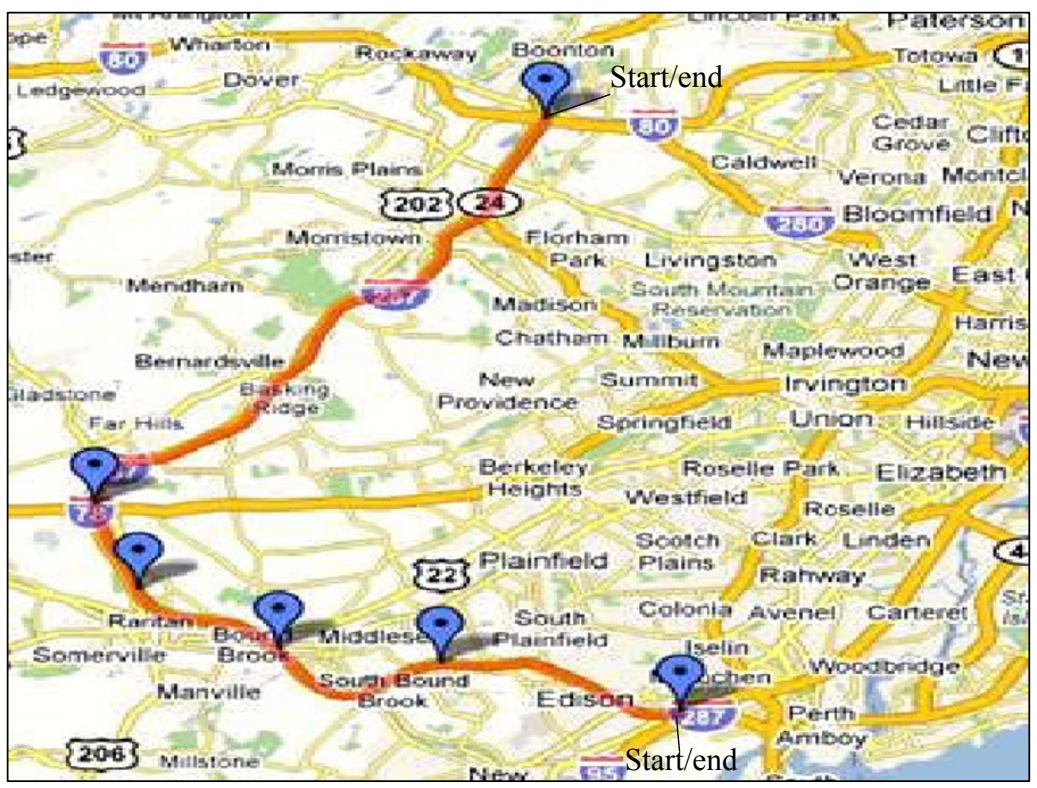

Fig. 2 I-287 \& TRANSMIT reader locations 


\section{Statistical indices}

To evaluate the difference in travel time data collected by different devices, the travel times were converted to vehicle speed values for each link in order to be evaluated by the following statistical measures:

(1) Standard error of the mean (SEM)

SEM, denoted as $\sigma_{\mathrm{SEM}}$ is defined as the standard derivation of the calculated error, or the difference between the travel time data collected by technologies to be evaluated (i.e., Bluetooth, INRIX, and TRANSMIT) and the ground truth data (collected by Co-Pilot devices), divided by the square root of the number of data $N$ collected during the evaluation time period $T$. If the duration of a data collection time is of length $\Delta$, then the number of time intervals, and therefore data points during the evaluation time period is

$$
N=T / \Delta \text {. }
$$

Let $c_{t}$ and $v_{t}$ denote the estimated speeds based on the Co-Pilot data and the data from other sources (e.g., INRIX, Bluetooth sensors, TRANSMIT readers), respectively during some time interval $t$. The corresponding error is $e_{t}=v_{t}-c_{t}$.

(2) Speed error bias (SEB)

$\mathrm{SEB}$, denoted as $e_{\mathrm{SEB}}$ is defined as the sum of the speed errors, (i.e., the difference between the estimated speeds based on the travel time data collected by Bluetooth, INRIX, and TRANSMIT and the data collected by Co-Pilot devices), divided by the number of data points $(N)$ taken for a given evaluation time period, denoted as $T$. Thus,

$$
e_{\mathrm{SEB}}=\frac{1}{N} \sum_{t=1}^{N} e_{t} .
$$

Then, the sample standard derivation of speed error is

$$
\sigma=\sqrt{\frac{\sum_{t=1}^{N}\left(e_{t}-\bar{e}_{t}\right)^{2}}{N-1}} .
$$

Hence, SEM can be derived from the sample standard derivation of the speed error (from Eq. 2) by dividing by the total number of intervals within the evaluation period:

$$
\sigma_{\mathrm{SEM}}=\frac{\sigma}{\sqrt{N}} .
$$

If the data are assumed to be normally distributed, the sample mean and standard error can be used to calculate approximate confidence intervals $\left(c_{i}\right)$ for the mean. The following expression can be used to calculate the upper and lower $95 \%$ confidence limits,

$$
c_{i}=\left[\bar{e}_{t}-1.96 \times \sigma_{\mathrm{SEM}}, \bar{e}_{t}+1.96 \times \sigma_{\mathrm{SEM}}\right],
$$

where $\bar{e}_{t}$ is the sample mean, and $\sigma_{\mathrm{SEM}}$ is the standard error of the sample mean.
(3) Average absolute speed error (AASE)

AASE, denoted as $e_{\mathrm{AASE}}$, is defined as the sum of absolute speed errors, (i.e. the difference between the estimated speeds based on the travel time data collected by Bluetooth, INRIX, and TRANSMIT and the data collected by CoPilot devices), divided by the number of data points $N$ taken for a given evaluation time period $T$. Thus,

$$
e_{\mathrm{AASE}}=\frac{1}{N} \sum_{t=1}^{N}\left|e_{t}\right| \text {. }
$$

\section{Analysis of travel time data}

With the equations formulated above, the statistical measures - including SEM, AASE and SEB-were calculated based on two groups of datasets for analysis.

The impact of link length and traffic conditions (e.g., congestion vs. un-congestion) on travel time estimation are assessed based on the observed data.

\subsection{Analysis of INRIX and Bluetooth vs. Co-Pilot travel time data}

Figs. 3 and 4 illustrate the relationship of speed versus time on links 1 and 3 of I-287 northbound, respectively. The traffic condition shown in Fig. 3 is quite stable, while that shown in Fig. 4 indicates significant change in travel time. To have an in-depth comparison of the INRIX, Bluetooth and Co-Pilot data, a statistical analysis is performed. The statistical measures, such as the SEM, AASE, and SEB are analyzed and summarized in Table 1. Compared to the INRIX data, in general, the speed estimation with the Bluetooth data is closer to the Co-Pilot data, because the SEM, AASE and SEB are smaller. The SEB of the INRIX data is 2.84 MPH, indicating that the INRIX data underestimated the speed detected by the probe vehicles.

On link 1, the travel speed has relatively small variations on both directions over the study time period. By comparing the trend line of different datasets, the curve of Bluetooth mean looks more adaptive to variations of the Co-Pilot data, while the curve of INRIX mean is more flat instead.

On link 3, the travel speed experienced severe variations on northbound over the study time period. The Bluetooth mean data has better fit to the Co-Pilot data. Though the INRIX data can still reflect the variations of the speed data, an obvious time lag was observed in the INRIX data, especially during the speed transition period.

Table 1 provides the statistics of links $1-4$ during the study period, which matches up with the conclusions based on the observation of Figs. 3-6. In general, Bluetooth data could generate better estimates of the traffic speed compared with INRIX data. The reason might be 
due to the static nature of the INRIX. INRIX data was extracted based on archived historical database, while Bluetooth data was collected in the same time period when Co-Pilot data was collected.

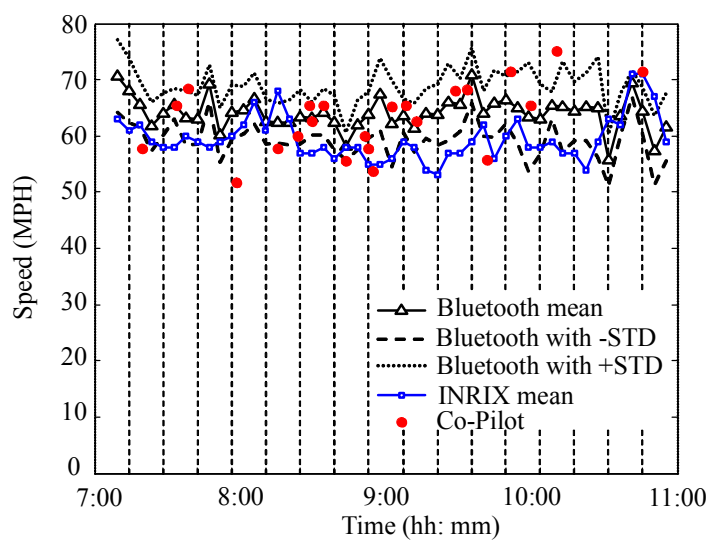

Fig. 3 Speed vs. time on link 1 of I-287 northbound

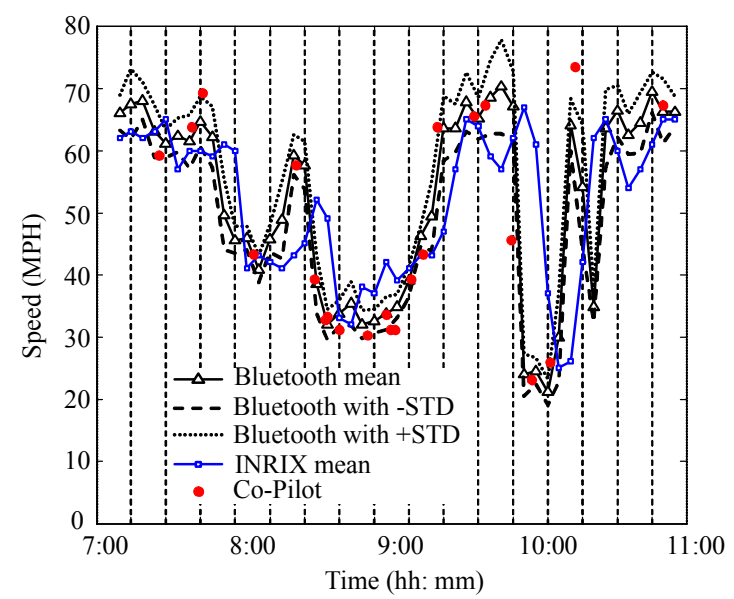

Fig. 4 Speed vs. time on link 3 of I-287 northbound

Table 1 Error comparison between Bluetooth, INRIX and Co-Pilotdata

(MPH)

\begin{tabular}{|c|c|c|c|c|c|c|c|}
\hline \multirow{2}{*}{$\begin{array}{l}\text { Link } \\
\text { No. }\end{array}$} & \multirow{2}{*}{$\begin{array}{l}\text { Type of } \\
\text { devices }\end{array}$} & \multicolumn{2}{|c|}{ SEM } & \multicolumn{2}{|c|}{ AASE } & \multicolumn{2}{|c|}{ SEB } \\
\hline & & $\mathrm{N}$ & $\mathrm{S}$ & $\mathrm{N}$ & $\mathrm{S}$ & $\mathrm{N}$ & $\mathrm{S}$ \\
\hline \multirow{2}{*}{1} & Bluetooth & 1.2 & 1 & 4.35 & 4.3 & 0.62 & -2.14 \\
\hline & INRIX & 1.5 & 1.3 & 6.78 & 5.1 & -3.6 & -3.49 \\
\hline \multirow{2}{*}{2} & Bluetooth & 1.3 & 1.2 & 5.25 & 4.6 & 2.3 & 2.52 \\
\hline & INRIX & 1.5 & 1 & 5.98 & 3.3 & -1.9 & -0.85 \\
\hline \multirow{2}{*}{3} & Bluetooth & 1.6 & 1.3 & 4.92 & 4.9 & 1.45 & -1.16 \\
\hline & INRIX & 3.5 & 1.2 & 11.2 & 4.9 & 2.1 & -3.76 \\
\hline \multirow{2}{*}{4} & Bluetooth & 1.1 & 1.4 & 4.15 & 4.8 & -0.6 & -0.98 \\
\hline & INRIX & 1.1 & 1.3 & 6.7 & 7.2 & -5.6 & -6.14 \\
\hline \multirow{2}{*}{ All } & Bluetooth & \multicolumn{2}{|c|}{0.46} & \multicolumn{2}{|c|}{4.65} & \multicolumn{2}{|c|}{0.32} \\
\hline & INRIX & \multicolumn{2}{|c|}{0.66} & \multicolumn{2}{|c|}{6.52} & \multicolumn{2}{|c|}{-2.84} \\
\hline
\end{tabular}

\subsection{Analysis of TRANSMIT vs. Co-Pilot travel time data}

Figs. 5 and 6 illustrate the relationship between speed and time of link 1 southbound and link 2 northbound on I-287, respectively, based on the data collected by TRANSMIT readers and Co-Pilot devices on April 9, 2009.

Fig. 5 shows that the probe vehicle did not experience the speed variation estimated by TRANSMIT on I-287 southbound. It might be the small sample size of probe vehicle that caused the missing of the speed variation.

As shown in Fig. 6, it was found that the travel speed experienced by probe vehicle fitted well with the speed estimated by TRANSMIT on link 2 of northbound I- 287 . The statistical measures are summarized in Table 2 to conclude the fitness of TRANSMIT data to Co-Pilot data for all links of I-287. The difference between speeds estimated by TRANSMIT and Co-Pilot devices was greater on link 1. It was also found that the link length on the studied links on I-287 did not affect the accuracy of speed estimation of TRANSMIT.

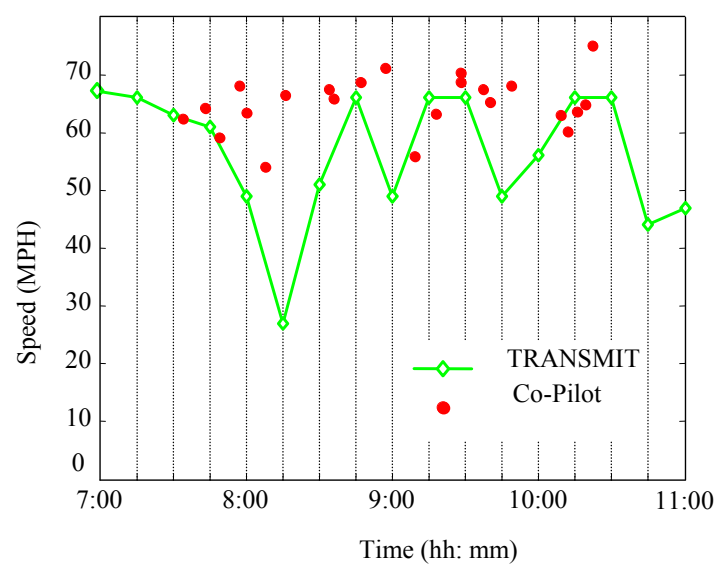

Fig. 5 Speed vs. time on link 1 of I-287 southbound

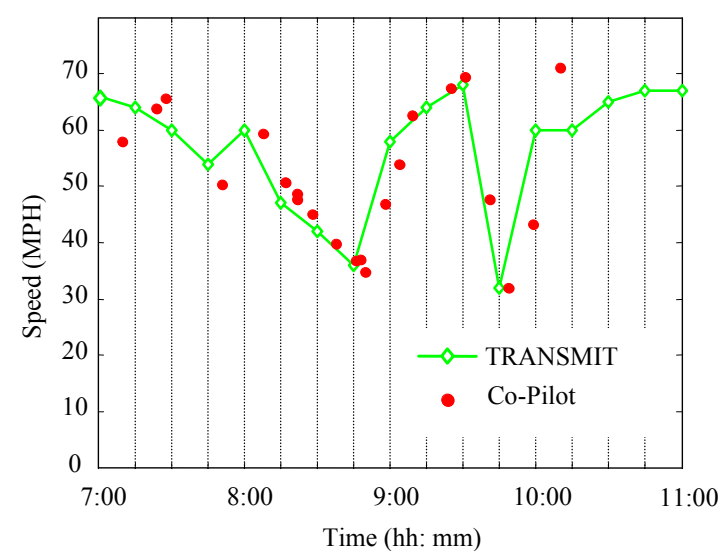

Fig. 6 Speed vs. time on link 2 of I-287 northbound 
Table 2 Error comparison of TRANSMIT vs. Co-Pilot data $(04 / 09 / 2009)$

(MPH)

\begin{tabular}{|c|c|c|c|c|c|c|c|}
\hline \multirow{2}{*}{$\begin{array}{l}\text { Link } \\
\text { No. }\end{array}$} & \multirow{2}{*}{$\begin{array}{l}\text { Link length } \\
\text { (miles) }\end{array}$} & \multicolumn{2}{|c|}{ SEM } & \multicolumn{2}{|c|}{ AASE } & \multicolumn{2}{|c|}{ SEB } \\
\hline & & $\mathrm{N}$ & $\mathrm{S}$ & $\mathrm{N}$ & $\mathrm{S}$ & $\mathrm{N}$ & $S$ \\
\hline 1 & 6.78 & 1.55 & 1.97 & 4.75 & 7.41 & 1.14 & -6.59 \\
\hline 2 & 5.79 & 1.43 & 1.13 & 4.29 & 4.76 & -0.41 & 2.80 \\
\hline 3 & 4.16 & 1.14 & 0.99 & 4.36 & 3.88 & 2.30 & 1.24 \\
\hline 4 & 3.78 & 1.11 & 1.31 & 4.51 & 5.66 & 2.86 & 3.17 \\
\hline 5 & 20.76 & 1.13 & 1.13 & 4.36 & 4.51 & 1.45 & 2.42 \\
\hline All & 41.27 & 0.58 & 0.69 & 4.45 & 5.26 & 0.99 & 0.57 \\
\hline
\end{tabular}

6.3. Analysis of travel time data (TRANSMIT, Bluetooth, INRIX vs. Co-Pilot)

To have a whole picture of the performance comparison of these various devices, the statistical measures of the data collected by these devices are put together in Table 3. It can be found that the performances of Bluetooth and TRANSMIT are better than that of INRIX. In addition, the performances of Bluetooth and TRANSMIT are quite similar, except that TRANSMIT overestimates the speed a little more.

Table 3 Error comparison of TRANSMIT, Bluetooth, INRIX vs. Co-Pilot data(04/09/2009)

(MPH)

\begin{tabular}{ccccc}
\hline Link No. & $\begin{array}{c}\text { Link length } \\
\text { (miles) }\end{array}$ & SEM & AASE & SEB \\
\hline TRANSMIT & 41.27 & 0.45 & 4.87 & 0.78 \\
Bluetooth & & 0.46 & 4.65 & 0.32 \\
INRIX & & 0.66 & 6.52 & -2.84 \\
\hline
\end{tabular}

This section summarizes the findings based on the analytical results with the data of INRIX, Bluetooth sensors, and TRANSMITS readers against the data collected by vehicle probes carrying Co-Pilot devices. To have a fair comparison of the accuracy of the INRIX, Bluetooth, and TRANSMIT data, this analysis was conducted in two parts: INRIX and Bluetooth data vs. CoPilot data and TRANSMIT data vs. Co-Pilot data, since the locations of TMC is different from the locations of TRANSMIT readers.

(1) The travel speed estimated with the Bluetooth data is closer to the speeds of vehicle probes compared to the INRIX data (see Table 2 and Table 3).

(2) The travel speed estimated with the INRIX data is biased with negative SEB.

(3) The travel speed experienced by probe vehicles fitted well with the speed estimated with TRANSMIT, except on link 1 of I-287 southbound. The probe vehicles seemed not to catch the speed variation reported by TRANSMIT.
(4) The link length on the studied links on I-287 did not affect the accuracy of speed estimation of TRANSMIT.

There is a data latency found in the INRIX data on north bound link 3, I-287 (see Fig. 4). This latency issue should be further investigated. The potential reasons might be but not be limited to, (1) inconsistency of speed estimation by time period and sample selection process, (2) delay in transmitting GPS data to satellite, cleaning and processing data, and populating results, and (3) biased samples ( $\%$ of trucks). Note that the Bluetooth and Co-Pilot data were not calculated in real time.

The INRIX data in 2009 seemed offer greater deviation to the ground truth data comparing the Bluetooth and TRANSMIT data, which could be affected by its data source, mainly commercial fleets, and the sufficiency of samples size. In order to provide a broader coverage on USA roadway network, INRIX data are extracted and generated based on GPS-enabled vehicles and mobile devices, traditional road sensors, etc. The historical data and data from commercial vehicles are all aggregated with the real-time data based on its "data fusion engine". Therefore, it inevitably led to certain biases that degraded the accuracy of its estimation under certain conditions. Both TRANSMIT and Bluetooth data are generated based on the same vehicle re-identification method that requires sets of detectors mounted along the road. A unique serial number for a device in the vehicles is detected at different locations, thus travel time and speed can be calculated. This process is done using the MAC addresses from Bluetooth devices, while TRANSMIT system uses the radio-frequency identification (RFID) serial numbers from the ETC transponders. TRANSMIT requires shorter range for signal capture compared with Bluetooth system. Overall Bluetooth and TRANSMIT system demonstrated the higher accuracy and reliability than INRIX data.

\subsection{Comparison of TRANSMIT and Co-Pilot data under incident condition}

During the study period (e.g. April 6, 2009-April 17, 2009), 21 incidents on I-287 were observed and reported. Four records of the reported incidents occurred in the studied area between 7:00-10:00 A.M. The incidents were observed on two different days (i.e., April 7 and April 16, 2009). However, Bluetooth and INRIX data (e.g. 7:00-10:00 A.M.) are not available for the period between April 6 and April 8 due to severe weather condition and unavailable INRIX data. Therefore, the analysis of the impact of incidents on travel time was conducted using Co-Pilot and TRANSMIT data. Fig. 7 is a time-space diagram for a probe vehicle that experienced 
an incident on link 2 of southbound I-287 on April 16, 2009. The $X$ axis represents the vehicle's location by mile post, and the $Y$ axis represents the time at which the vehicle is located.

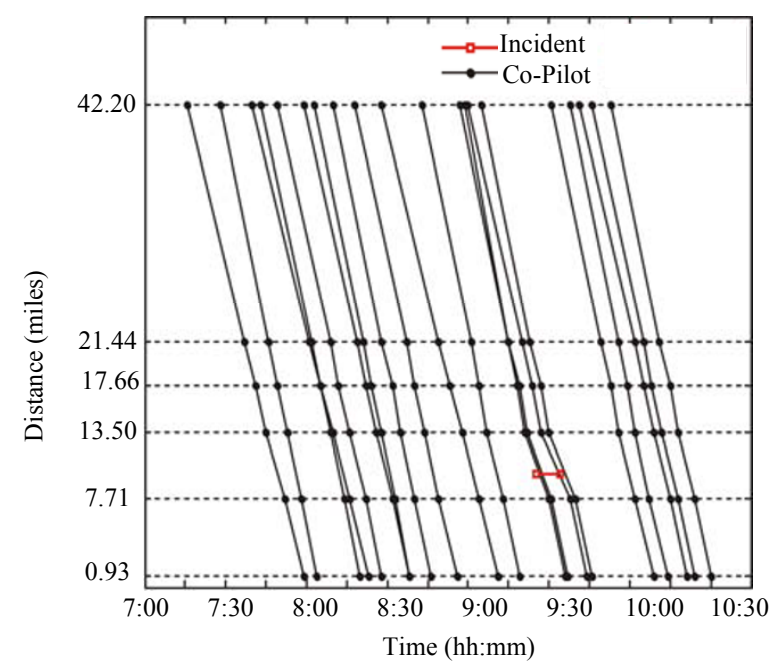

Fig. 7 Distance vs. time (incident \#3, 04/16/09 SB)

Thus, the curve represents the trajectory of the probe vehicle and its slope stands for the traveling speed. The red line indicates the time period when the incident occurred, from 9:25 A.M. to 9:45 A.M. It can be observed that the speed decreased during that time period. Fig. 8 illustrates the speed of the TRANSMIT data and CoPilot data, and the speed decrease during the same time period on link 2 can also be observed.

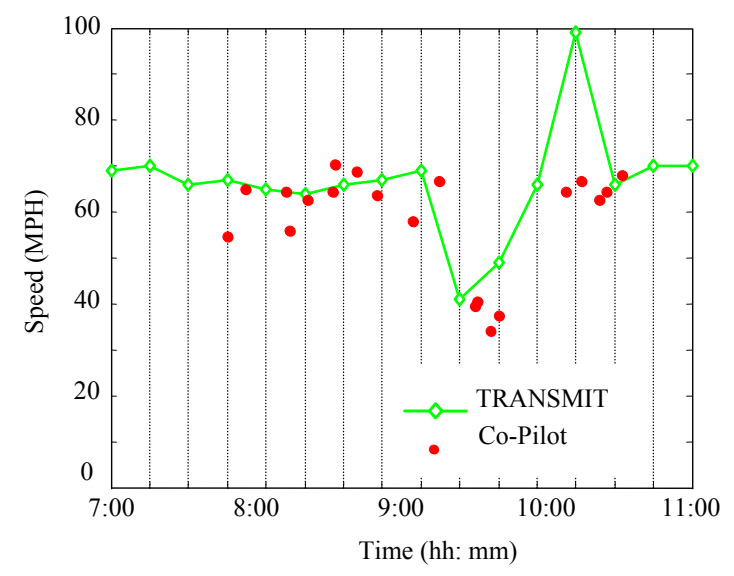

Fig. 8 Speed vs. time (incident \#3, 04/16/09 SB)

From Table 4, the following can be found:

(1) Both the data collected by probe vehicles and by TRANSMIT can reveal the change of traffic speed if the traffic condition is influenced by the reported incidents, for example the reported incident from 9:25 A.M. to 9:34 A.M. on April 16 2009, as shown in Figs. 7 and 8.

(2) Based on Table 4, it can be found that the estimate with TRANSMIT data is a little bit worse under incidents, compared to the case without incident, due to the larger SEM and AASE. The SEB under incidents is smaller (-0.05 MPH compared to $1.30 \mathrm{MPH})$ because of the offset effect between 7.67 MPH and -7.21 MPH.

(3) There are abrupt speed changes observed around 10:00 AM in Fig. 8 based on the TRANSMIT data. However, the speed estimates based on probe vehicles within this time interval do not demonstrate these abrupt changes. Further investigation shall be conducted.

Table 4 Error comparison of TRANSMIT vs. Co-Pilot data with and without incidents

(MPH)

\begin{tabular}{ccccccccc}
\hline \multirow{2}{*}{$\begin{array}{c}\text { Incident } \\
\text { No. }\end{array}$} & \multicolumn{2}{c}{ SEM } & & \multicolumn{2}{c}{ AASE } & & \multicolumn{2}{c}{ SEB } \\
\cline { 2 - 5 } \cline { 6 - 8 } incident & $\begin{array}{c}\text { Without } \\
\text { incident }\end{array}$ & $\begin{array}{c}\text { With } \\
\text { incident }\end{array}$ & $\begin{array}{c}\text { Without } \\
\text { incident }\end{array}$ & $\begin{array}{c}\text { With } \\
\text { incident }\end{array}$ & $\begin{array}{c}\text { Without } \\
\text { incident }\end{array}$ \\
\hline 1 & 0.81 & 1.13 & 3.34 & 4.76 & & 1.14 & 2.80 \\
2 & 2.79 & 1.13 & 8.81 & 4.76 & 7.67 & 2.80 \\
3 & 1.18 & 1.13 & 3.55 & 4.36 & -0.82 & 1.45 \\
4 & 2.40 & 1.45 & 8.37 & 4.29 & -7.21 & -0.41 \\
All & 1.12 & 0.72 & 6.01 & 4.48 & -0.05 & 1.30 \\
\hline
\end{tabular}

\section{Conclusions}

The mean speed estimates in INRIX and Bluetooth data are provided on a regular 5-minute basis. The daily study period using Co-Pilot devices was divided into twelve 15-minute time intervals from 7:00 A.M. to 10:00 A.M., with two data collectors dispatched per time interval. An analysis of differences in data between INRIX, Bluetooth and Co-Pilot data showed that speed estimation using Bluetooth technology is closer to the Co-Pilot data compared to the INRIX data. The SEBs of the INRIX data are consistently lower, indicating that the INRIX data underestimated the speed comparing to "ground truth" data collected by GPS probes.

Regarding to TRANSMIT and Co-Pilot data, it was found that the "ground truth" travel speeds in general fit well with speeds reported by TRANSMIT. It was also found that the link length of the study link I-287 did not affect the accuracy of reported speeds by TRANSMIT. Both "ground truth" and TRANSMIT speeds were influenced by incidents. The accuracy of speeds reported by TRANSMIT was slightly worse under non-recurring congestion, such as incidents. However, the accuracy of speed estimates with either Bluetooth or TRANSMIT data outperformed that with the INRIX data. In addition, the accuracy of speed estimates with both Bluetooth and TRANSMIT data were similar, except that TRANSMIT slightly overestimated travel speed of the study I-287.

Overall, this study successfully tested the feasibility of utilizing different floating car technologies for traffic 
data collection. The availability and consistency for preparing different FCDs are described. The accuracy of each travel time estimation method is validated and compared. The in-depth analysis and results in the study can be concluded as follows:

(1) The accuracy of the INRIX and Bluetooth data vs. the Co-Pilot data and TRANSMIT data vs. Co-Pilot data were evaluated.

(2) The travel speed estimated with Bluetooth sensors is closer to the ground true speed when compared to the INRIX data.

(3) The ground true speed seems to fit well with the TRANSMIT data.

(4) The link length on the study I-287 seemed no impact on the accuracy of speed estimation with TRANSMIT.

(5) TRANSMIT speed estimates were slightly closer to the Co-Pilot data under regular traffic conditions than in the event of an incident.

(6) Both Co-Pilot data and TRANSMIT data reveal speed variations as an incident occurs.

To further investigate the causes of discrepancy in estimated travel times based on the studied floating car technologies, future research shall focus on:

(1) Analyzing INRIX data and methods to process the data to identify the potential reasons that may cause biased estimation.

(2) Analyzing Bluetooth data and the detection rates under various conditions (i.e., traffic, weather, geometry, and spacing between sensors).

(3) Analyzing the accuracy of TRANSMIT data considering the link length and those links with rest areas.

\section{Acknowledgements}

The authors would like to acknowledge the support of Drs. K. Mouskos (City University of New York), M. Boile (Rutgers University, the State University of New Jersey), and A. Kornhauser (Princeton University), who offered valuable comments while collecting and processing the data of this study.

\section{References}

[1] C. Dalaff, R. Reulke, A. Kroen, et al., A traffic object detection system for road traffic measurement and management, http://citeseerx.ist.psu.edu/viewdoc/summary? doi=10.1.1.113.596, 2003.
[2] M. Chen, S. Chien, X. Liu, et al., Application of AVL/APC data support system, In: The 82th TRB Annual Meeting, Preprint CD-ROM, Washington DC: Transportation Research Board, 2003.

[3] J. Wright, J. Dahlgren, Using vehicles equipped with toll tags as probes for providing travel times, California PATH Working Paper, 2001.

[4] M.D. Fontaine, A.P. Yakala, B.L. Smith, Final contract report: probe sampling strategies traffic monitoring systems based on wireless location technology, Virginia Transportation Research Council, 2007.

[5] K. Oberstein, Collection and use of floating car data: experiences from Berlin, In: ITS-World Congress, 1997, Berlin: ITS, 1997

[6] U. Fastenrath, Floating car data on a larger scale, In: ITS-World Congress, 1997, Berlin: ITS, 1997.

[7] M. Lädke, W. Huber, Extended mobile data collection for immediate driver warning, VDI- Conference for Electronic Systems for Vehicles; Baden-Baden, Germany, 1998.

[8] B. Hellinga, L. Fu, Reducing bias in probe-based arterial link travel time estimates, Transportation Research Part C, 2002, 10(4): 257-273.,

[9] P. Loustau, V. Grasset, C. Morency, et al., Comparing fl oating car data and carsharing GPS data for travel time a ssessment, http://intranet.imet.gr/Portals/0/UsefulDocuments/documents/02030.pdf, 2010.

[10] B. Smith, H. Zhang, M. Fontaine, et al., Final report of ITS center project: cell-phone probes as an ATMS tool, Charlottesville: University of Virginia Center for Transportation Studies, 2003.

[11] B. Kerner, C. Demir, R. Herrtwich, et al., Traffic state detection with floating car data in road networks, In: IEEE International Conference on Intelligent Transportation Systems, Konolige, 2005.

[12] G. Rose, Mobile phones as traffic probes: practices, prospects and issues, Transport Reviews, 2006, 26(3): 275-291.

[13] E. Brockfeld, S. Lorkowski, P. Mieth, et al., Benefits and limits of recent floating car data technology: an evaluation study, In: The 11th WCTR Conference, Berkeley, 2007.

[14] G. Leduc, Road traffic data: collection methods and appl ications, JRC Technical Notes, http://ftp.jrc.es/EURdoc/ JRC47967.TN.pdf, 2008.

[15] K. Mouskos, E. Niver, L. Pignataro, TRANSMIT system evaluation, Final Report, Newark: New Jersey Institute of Technology.

(Editor: Yao ZHOU) 